\title{
Depresion en pacientes con alteraciones del tiroides
}

\author{
Ljiljana Radanović-Grgurić, mr. sc., MD, \\ Médico Psiquiatra* \\ Pavo Filaković, PhD, MD, Profesor \\ Asociado de Psiquiatria* \\ Jelena Barkić, mr. sc., Psicóloga* \\ Nikola Mandić, PhD, MD, Profesor de \\ Psiquiatria* \\ Ivan Karner, PhD, MD, Profesor de \\ Fisiopatología** \\ Juraj Smoje, mr. sc., MD** \\ * Hospital Clínico de Osijek; \\ ** Medicina Nuclear Hospital Clínico de \\ Osijek; \\ CROACIA
}

\begin{abstract}
RESUMEN - Nuestro estudio fue realizado en un grupo de 53 mujeres con disfunción tiroidea y 28 mujeres con depresión mayor. Empleamos la Escala de la Depresión de Hamilton, la Escala de Autoevaluación de la Depresión de Zung y la Escala sobre la Impresión Clínica Global. Los resultados del estudio demuestran que la mayoría de los pacientes con disfunción tiroidea se mostraron clínicamente significativos en cuanto al trastorno depresivo. Los episodios depresivos son más frecuentes en pacientes con hipotiroidismo que en aquellos que presentan hipertiroidismo. Era menos grave en pacientes con disfunción tiroidea que en aquellos que habían sido diagnosticados de depresión mayor unipolar. Los pacientes con hipertiroidismo tenían menos síntomas graves en cuanto a la inhibición y los pacientes con hipotiroidismo tenían menos síntomas graves de agitación que los pacientes con depresión mayor unipolar. Podemos concluir diciendo que los pacientes con disfunción tiroidea presentan un riesgo potencial para los trastornos depresivos por lo que el diagnóstico y tratamiento se recomienda con el fin de evitar el riesgo de la cronicidad.
\end{abstract}

\section{Introducción}

Los trastornos depresivos son uno de los problemas de salud más significativos de las sociedades desarrolladas. Más de 120 millo- nes se encuentran afectas de depresión lo que viene a ser el 3\% de la población mundial. Eso significa que entre 10 a 20 millones de personas han tenido al menos un episodio depresivo en sus vidas (Cancro 1985, Sarto- 
rius 1986). Solamente un pequeño número de las personas afectas reciben tratamiento psiquiátrico. La mayor parte de las personas afectas no son diagnosticadas y los síntomas quedan camuflados por patologías orgánicas. La consecuencia es que no van a recibir tratamiento específico para la depresión.

La correlación de la función tiroidea con determinadas características psicológicas fue puesta de manifiesto en las primeras descripciones sobre las disfunciones tiroideas (Parry 1825, Clinical society of London 1888). Se trataba de manifestaciones tales como tensión, irritabilidad, delusiones e histeria. Descripciones posteriores centradas en los aspectos depresivos, así como el empleo de tests psicológicos estandarizados y escalas psiquiátricas, llevaron consigo el que las observaciones se hicieran más objetivas (Bauer et al. 1987, Joffe et al. 1992, Prange et al. 1974).

Algunos artículos psiquiátricos nos han mostrado la correlación que existe entre las formas subclínicas del hipotiroidismo y los trastornos afectivos (Gold et al. 1981, Cowdray et al. 1983, Wenzel et al. 1974). Otros autores han observado la correlación existente entre el hipotiroidismo y la depresión y/o la hipomania o el trastorno bipolar (Ckeekly 1978, Peake 1981, Villani y Wertzel 1979). En los últimos 30 años hay un mayor interés por conocer mejor todo lo relacionado acerca de las relaciones entre la función tiroidea y los trastornos psiquiátricos, así como sobre sobre el metabolismo de las hormonas tiroideas, el funcionamiento del eje hipotálamo-hipófisistiroides y los modalidades de neurotransmisión en el SNC (Daniels y Martín 1991, Duval et al. 1999, Jackson 1983, Lucke et al. 1977, Musselman y Nemeroff 1986, Tuomisto y Mannisto 1985, Wartofsky e Ingbar 1991) y en fin, sobre todo lo relativo a los nuevos modelos neurobiológicos de la depresión (Gold et al. 1989, Prange et al. 1974, Schildkraut 1965, Schildkraut et al. 1985, Schmaus
1992, Vander Kar 1989, Whybrow y Prange Jr. 1981). Existen publicaciones acerca de las distintas alteraciones presentes en los tests hormonales que evalúan el funcionamiento del eje hipotálamo-hipófisis-tiroides en la depresión: disminución de la secreción de la TSH ante la estimulación mediante la TRH (Amsterdam et al. 1996, Extein et al. 1982, Hofmann y Schrotter 1999, Muller y Bonning 1988, Prange et al. 1972), y sobre las alteraciones de la concentración en sangre de hormonas tiroideas en el caso de la depresión (Bartalena et al. 1990, Copolov y Rubin 1987, Dorn et al. 1986, Kirkegaard et al. 1990, Kjelmann et al. 1984, Muller y Bonning 1988, Rubin et al. 1987). Algunos experimentos terapéuticos han señalado los beneficiosos efectos de las hormonas tiroideas asociadas al tratamiento antidepresivo en el caso de las depresiones resistentes (Earle 1970, Joffe 1998, Lasser y Baldessarini 1997, Loosen y Prange 1982, Moreau et al. 2001, Nakamura y Nomura 1992, Prange et al. 1969). La realidad es que el normal funcionamiento de la glándula tiroidea es un prerequesito para el normal funcionamiento cerebral, junto con las anteriores consideraciones, en torno a la correlación existente entre el funcionamiento del eje hipotálamo-hipófisis-tiroides, la depresión y los trastornos afectivos. La esencia de esta correlación, no se termina de aclarar, y ello ha sido el motivo de la realización de muchos ensayos clínicos. El fin de nuestro estudio ha sido el determinar la frecuencia de la depresión en pacientes con alteración de la función tiroidea, así como la gravedad de los síntomas depresivos.

\section{Material y método}

El estudio incluye 53 pacientes de sexo femenino con una edad media de 47,3 años de 
edad con disfunción tiroidea (30 con hipertiroidismo y 23 con hipotiroidismo), y 28 mujeres con trastorno depresivo unipolar (definidos de acuerdo con el Manual Diagnóstico Estadístico de los Trastornos Mentales, el DSM-IV), con una edad media de 51,5 años. Todos los procedimientos utilizados en el estudio llevado a cabo contaron con la adecuada comprensión y con el consentimiento informado por parte de los sujetos partícipes en esta investigación. Estos fueron entrevistados por un psiquiatra, antes de empezar con un tratamiento específico. La escala de Hamilton para la Depresión y la Escala de Autoevaluación de Zung se emplearon para discriminar aquellos pacientes que padecían de depresión, empleándose además la Escala de Impresión Clínica Global para determinar la gravedad del trastorno mental. Se considero que la clínica era significativa de trastorno depresivo en aquellos pacientes que puntuaban con al menos 17 en la Escala de Hamilton para la Depresión. El test de Chi-cuadrado se empleó para evaluar estadísticamente los valores de las escalas de depresión, mientras que la $\mathrm{T}$ de Student se empleó para estudiar las distintas hipótesis planteadas acerca de las medidas expresados como valores medios.
Las diferencias observadas se consideraron como estadísticamente significativas cuando se daban valores menores del 5 y del $1 \%$.

\section{Resultados}

De los 30 pacientes con hipertiroidismo, 17 cumplían los criterios de Trastorno de Depresivo, mientras que en el caso de los 23 pacientes con hipotiroidismo 16 padecían de Trastorno Depresivo. El diagnóstico se realizó utilizando los criterios diagnósticos del DSM-IV, tanto para el diagnóstico de Trastorno Depresivo Mayor como Menor. La sintomatología era significativa de Trastorno Depresivo cuando puntuaba por encima de 17 en la Escala de Hamilton para la Depresión. Posteriormente los pacientes con Trastorno depresivo fueron comparados con un grupo de pacientes con Depresión Mayor Unipolar.

Los grupos de pacientes difieren en la gravedad de la depresión y en las puntuaciones obtenidas en las escalas para la Depresión (tabla I, figura 1).

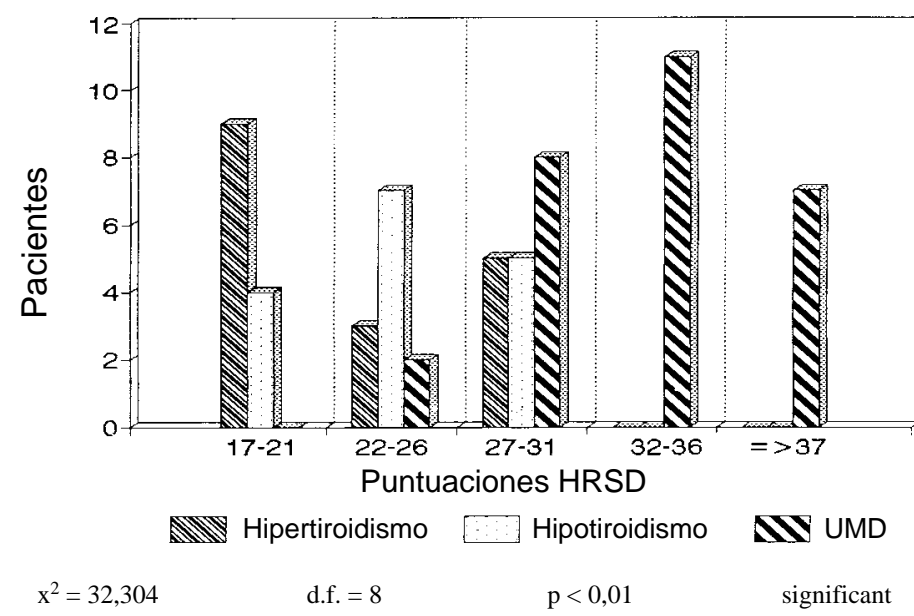

Figura 1. Comparación de las puntuaciones con la Escala para la Depresión de Hamilton (HRSD) de pacientes con hipertiroidismo, hipotiroidismo y depresión mayor unipolar (UMD). 
Tabla I

Puntuaciones medias en la Escala para la Depresión de Hamilton (HRSD) y la Escala Autoaplicada para la Depresión de Zung (SRDS) en pacientes con hipertiroidismo, hipotiroidismo y depresión mayor unipolar (UMD)

\begin{tabular}{llll} 
Diagnóstico & $\mathrm{N}$ & HRSD & \multicolumn{1}{c}{ SRDS } \\
\hline Hipertiroidismo & 17 & $23.12 \pm 4.34$ & $53.41 \pm 8.15$ \\
Hipotiroidismo & 16 & $24.69 \pm 3.89$ & $55.75 \pm 10.36$ \\
UMD & 28 & $33.25 \pm 5.36$ & $63.89 \pm 5.64$ \\
\hline
\end{tabular}

Índice SRDS:

41 - 47 Depresión media.

48 - 55 Depresión moderada a grave.

> 55 Depresión grave.

La valoración de la gravedad del trastorno mental se estableció mediante la Escala de Impresión Clínica Global, en la misma línea que se había establecido con para las escalas de depresión con una diferencia significativa $\left(x^{2}=25,438\right.$; d.f. $\left.=; p<0,01\right)$ (figura 2). Un análisis paralelo de los síntomas de trastorno depresivo y los resultados obtenidos mediante la Escala de Hamilton para la Depresión mostró que todos los pacientes habían perdi- do interés en el trabajo y tenían sintomatología física. La mayoría de los pacientes con disfunción tiroidea mostró signos de depresión y alteraciones del sueño. Los pacientes de los diferentes grupos mostraron diferentes manifestaciones depresivas como nos muestra la tabla II.

Los grupos de pacientes con hipertiroidismo, hipotiroidismo y Trastorno Depresivo
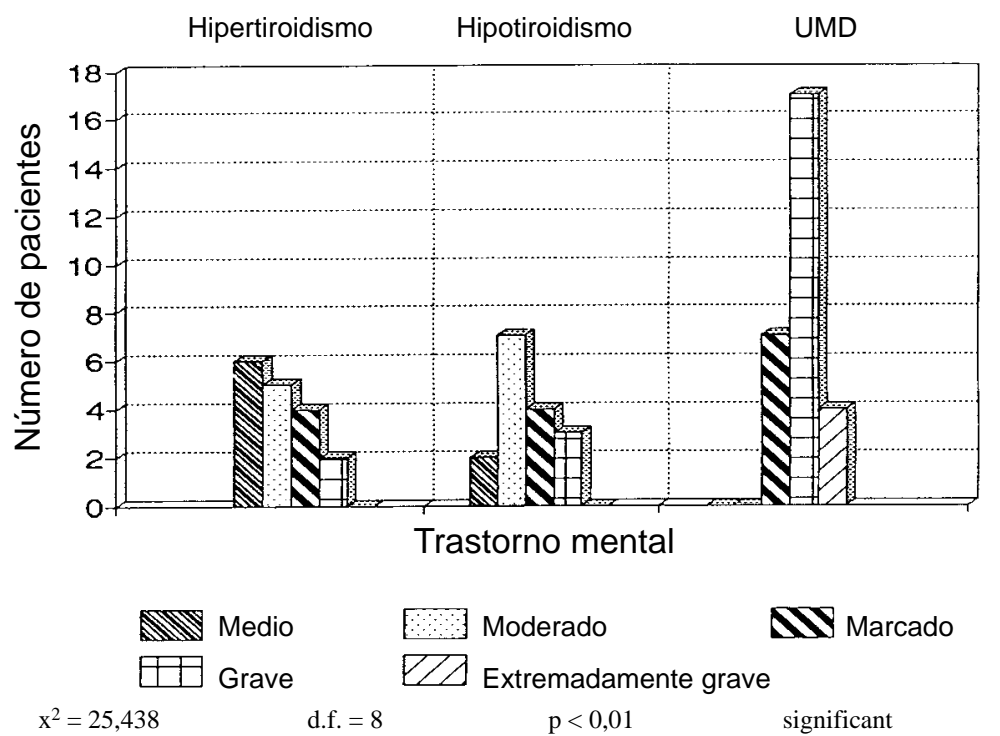

Figura 2. Intensidad del trastorno mental de acuerdo con la Impresión Clínica global en relación con el hipertiroidismo, el hipotiroidismo y la depresión mayor unipolar (UMD). 
Tabla II

Presencia de síntomas depresivos de acuerdo con Escala para la Depresión de Hamilton (HRSD) en grupos de pacientes con hipertiroidismo, hipotiroidismo y depresión mayor unipolar (UMD)

\begin{tabular}{|c|c|c|c|c|}
\hline \multirow{2}{*}{$\begin{array}{l}\text { Presencia de síntomas } \\
\text { de acuerdo con la HRSD }\end{array}$} & \multicolumn{4}{|c|}{ Hipotiroidismo } \\
\hline & \multicolumn{2}{|c|}{ Hipertiroidismo } & & UMD \\
\hline \multirow[t]{2}{*}{ Humor depresivo } & SI & 16 & 16 & 28 \\
\hline & NO & 1 & 0 & 0 \\
\hline \multirow[t]{2}{*}{ Sentimiento de culpa } & SI & 4 & 2 & 16 \\
\hline & NO & 13 & 14 & 12 \\
\hline \multirow[t]{2}{*}{ Suicidabilidad } & SI & 10 & 15 & 27 \\
\hline & $\mathrm{NO}$ & 7 & 1 & 1 \\
\hline \multirow[t]{2}{*}{ Problemas para dormir } & SI & 14 & 13 & 28 \\
\hline & NO & 3 & 3 & 0 \\
\hline \multirow[t]{2}{*}{ Sueño interrumpido } & SI & 16 & 16 & 28 \\
\hline & $\mathrm{NO}$ & 1 & 0 & 0 \\
\hline \multirow[t]{2}{*}{ Despertar temprano } & SI & 12 & 15 & 28 \\
\hline & NO & 5 & 1 & 0 \\
\hline \multirow{2}{*}{$\begin{array}{l}\text { Pérdida del interés por el trabajo } \\
\text { y las actividades }\end{array}$} & SI & 17 & 16 & 28 \\
\hline & NO & 0 & 0 & 0 \\
\hline \multirow[t]{2}{*}{ Inhibición psicomotora } & SI & 12 & 16 & 26 \\
\hline & NO & 5 & 0 & 2 \\
\hline \multirow[t]{2}{*}{ Agitación } & SI & 17 & 6 & 27 \\
\hline & NO & 0 & 10 & 1 \\
\hline \multirow{2}{*}{ Ansiedad psíquica } & SI & 17 & 12 & 28 \\
\hline & NO & 0 & 4 & 0 \\
\hline \multirow[t]{2}{*}{ Ansiedad somática } & SI & 17 & 15 & 26 \\
\hline & $\mathrm{NO}$ & 0 & 1 & 2 \\
\hline \multirow[t]{2}{*}{ Síntomas gastrointestinales } & SI & 7 & 12 & 26 \\
\hline & $\mathrm{NO}$ & 10 & 4 & 2 \\
\hline \multirow[t]{2}{*}{ Síntomas somáticos } & SI & 17 & 16 & 28 \\
\hline & NO & 0 & 0 & 0 \\
\hline \multirow[t]{2}{*}{ Síntomas genitales } & SI & 15 & 14 & 27 \\
\hline & $\mathrm{NO}$ & 2 & 2 & 1 \\
\hline \multirow[t]{2}{*}{ Hipocondríasis } & SI & 6 & 4 & 16 \\
\hline & NO & 11 & 12 & 12 \\
\hline \multirow[t]{2}{*}{ Pérdida de peso } & SI & 16 & 5 & 22 \\
\hline & NO & 1 & 11 & 6 \\
\hline \multirow[t]{2}{*}{ Introspección } & SI & 2 & 2 & 6 \\
\hline & $\mathrm{NO}$ & 15 & 14 & 22 \\
\hline
\end{tabular}

Mayor mostraron diferencias estadísticamente significativas con respecto a la gravedad de los síntomas manifestados como nos muestra las figuras 3, 4 y 5 .

Tres pacientes con hipertiroidismo y cuatro con hipotiroidismo habían presentado episodios depresivos previos, por lo que el episodio depresivo actual fue considerado como recurrente.

\section{Discusión}

Algo más de la mitad de los pacientes con hipertiroidismo y las dos terceras partes de los pacientes con hipotiroidismo incluidos en nuestro estudio presentaban sintomatología propia de un trastorno depresivo. Nuestros resultados parecen estar en la misma línea que los de los hallazgos actualmente 


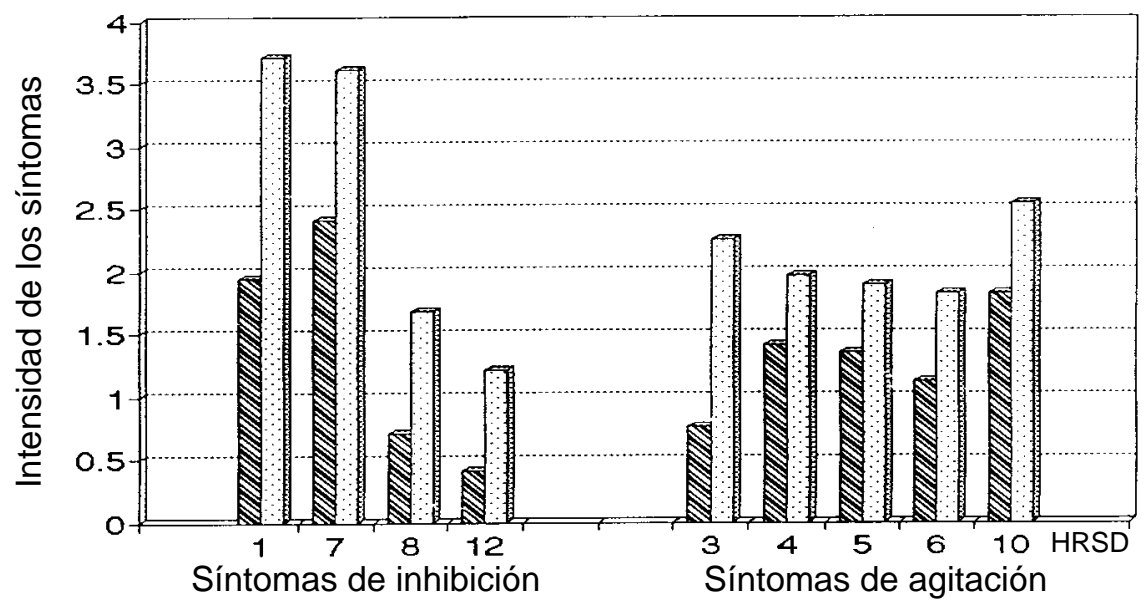

Hipertiroidismo UMD

HRSD:

1. Humor depresivo.

7. Pérdida de interés por el trabajo.

8. Inhibición psicomotora.

12. Síntomas gastrointestinales (pérdida de apetito y constipación).
3. Tendencias siucidas.

4. Problemas para dormirse.

5. Sueño interrumpido.

6. Despertar temprano.

10. Ansiedad psíquica.

Figura 3. Síntomas diferentes significativos de acuerdo con la Escala para la Depresión de Hamilton (HRSD) en el grupo de pacientes con hipertiroidismo y en el grupo de pacientes con depresión mayor unipolar (UMD).

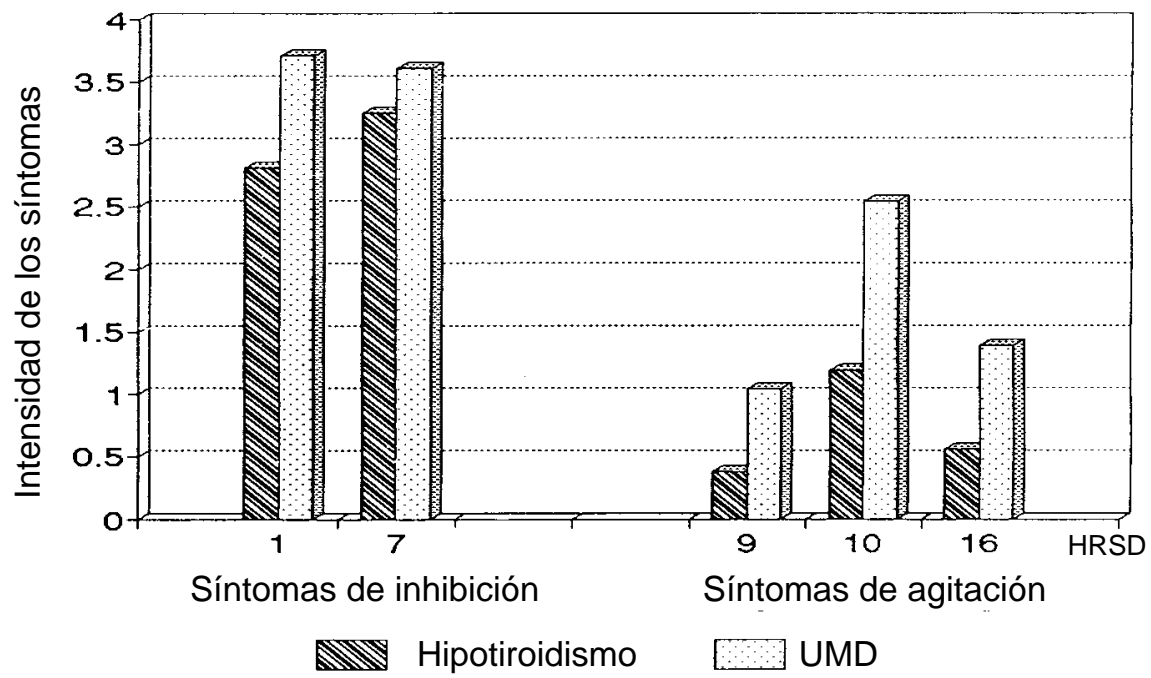

HRSD:

1. Humor depresivo.

9. Agitación.

7. Pérdida de interés por el trabajo.

10. Ansiedad psíquica.

16. Pérdida de peso.

Figura 4. Síntomas diferentes significativos de acuerdo con la Escala para la Depresión de Hamilton (HRSD) en el grupo de pacientes con hipotiroidismo y en el grupo de pacientes con depresión mayor unipolar (UMD). 


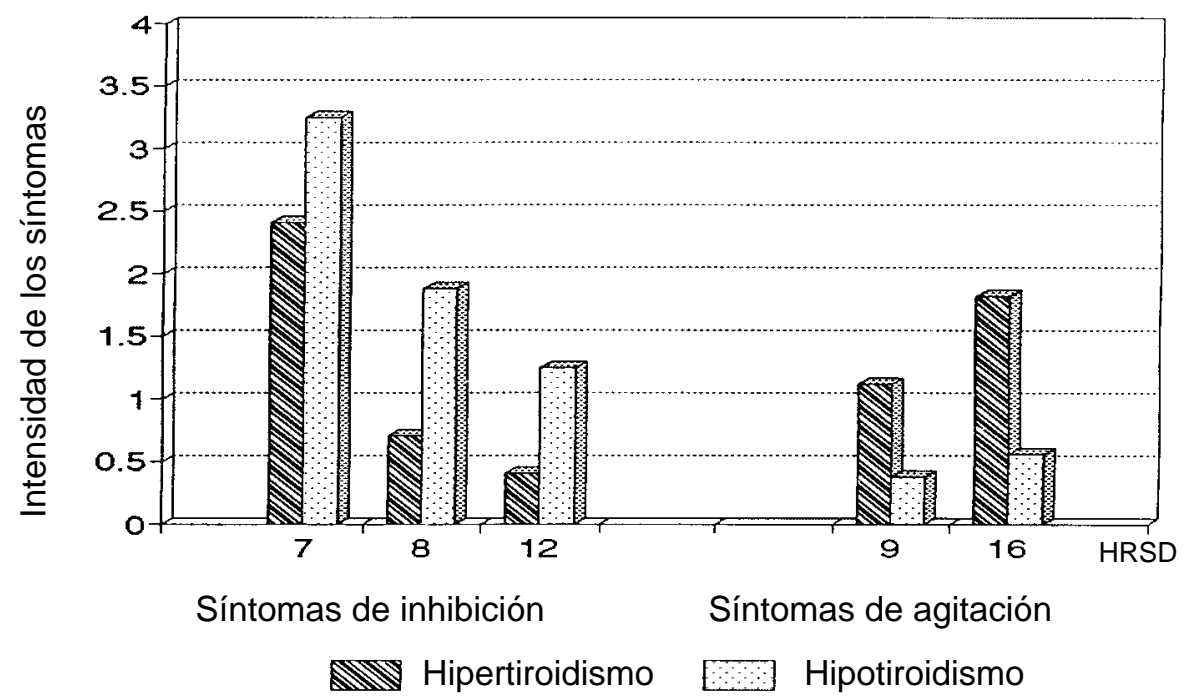

HRSD:

7. Pérdida interés por el trabajo.

8. Ihibición psicomotora.
12. Síntomas gastrointestinales (pérdida de apetito y constipación).

9. Agitación.

16. Pérdida de peso.

Figura 5. Síntomas diferentes significativos de acuerdo con la Escala para la Depresión de Hamilton (HRSD) en el grupo de pacientes con hipertiroidismo y en el grupo de pacientes con hipotiroidismo.

existentes. En 1962 Wilson et al. fueron los primeros en notificar manifestaciones depresivas en 15 de 26 pacientes diagnosticados de hipotiroidismo. En 1969 Whybrow et al. en 7 pacientes con hipotiroidismo, observó que 5 de ellos presentaban sintomatología depresiva y además uno de ellos incluso mostraba síntomas psicóticos. Estos autores señalaron como los pacientes con hipotiroidismo tenían altas puntuaciones en la Escala de Depresión del Inventario Multiaxial de Personalidad de Minnesota (MMPI). En la mayor parte de las escalas daban puntuaciones superiores a las obtenidas por los pacientes con hipertiroidismo.

En el caso de la BPRS, el total de las puntuaciones fue mayor en el caso de los pacientes con hipotiroidismo que las obtenidas en pacientes con hipertiroidismo. En 1980, Rockey y Gripe encontraron a un paciente con Depresión Mayor en un grupo de 14 pacientes con hipertiroidismo. En 1972 Jain et al. encontraron que de entre 30 pacientes con hipotiroidismo 13 estaban diagnosticados de trastorno depresivo de acuerdo con el Inventario de Depresión de Hamilton y Beck. Los pacientes con hipotiroidismo presentaron altas puntuaciones medias. Es especialmente llamativo como los pacientes con hipotiroidismo grave obtuvieron los mas bajos resultados en las escalas de evaluación.

En 1979, Mac Crimmon et al. estudiaron a 19 mujeres con hipertiroidismo empleando la Escala de Evaluación Psiquiátrica PSS y el MMPI. Los pacientes mostraron altas puntuaciones para la depresión y la ansiedad, y generalmente mostraron mas síntomas en comparación con un grupo de control sano. No obstante ello, los perfiles de las puntuaciones con el PSS no guardaban relación con ninguna entidad clínica específica. En cuanto al MMPI las mayores puntuaciones aparecían en relación con las escalas de depresión y de ansiedad. 
El análisis de los síntomas depresivos que se hicieron manifiestos y con diferencias significativas entre los grupos de pacientes ofrecieron características relevantes: los pacientes con hipertiroidismo mostraron síntomas de inhibición menos graves que los pacientes con cuadros depresivos mayores unipolares. Entre los grupos de pacientes con hipotiroidismo y con depresiones mayores, los síntomas de inhibición (humor depresivo y pérdida del interés por el trabajo) fueron mas graves, y aún menos entre los pacientes con hipotiroidismo. Los síntomas de agitación eran mas graves en los pacientes con Depresión Mayor, manifestándose con fuerte angustia, pérdida de peso y agitación. La comparación de la gravedad de los síntomas depresivos en pacientes con disfunción tiroidea mostró como los síntomas de inhibición prevalecían entre los pacientes con hipotiroidismo, quienes también experimentaron mas cuadros depresivos graves. Los síntomas de agitación prevalecían entre los pacientes con hipertiroidismo. Nuestras observaciones están en línea con los datos ya existentes: Whybrow et al. señalaron ya en el año 1969 como los pacientes con hipotiroidismo daban altas puntuaciones mediante la BPRS en los items relativos a la inhibición motora y al humor depresivo que lo que sucedía entre los enfermos con hipertiroidismo. Los pacientes hipertiroideos ofrecían en esa misma escala puntuaciones significativamente más altas en el ítem referente a la inquietud motora. Mediante el empleo del MMPI y la Clyde Mood Scale, estos mismos autores registraron altas puntuaciones en lo referente a síntomas tales como histeria y agitación entre los pacientes con hipertiroidismo, así como menores niveles depresivos en comparación con los pacientes hipotiroideos.
Nosotros podemos estimar que siete pacientes con disfunción tiroidea y trastornos depresivos manifiestos sufren de Trastornos Depresivo Recurrente. Pensamos igualmente que la disfunción tiroidea probablemente reduzca el umbral como para que se desencadene un episodio depresivo. Parecidas son las conclusiones derivadas de las investigaciones de Denicoff et al. desde 1990 y las de Haggerty et al. desde 1993.

La literatura internacional hace hincapié sobre la importancia de tratar el hipotiroidismo, especialmente el subclínico como actuación preventiva de cuadros depresivos que puedan aparecer. En 1992, Joffe y Levitt manifestaron que la prevalencia de los diferentes tipos de hipotiroidismo es probablemente mas alta entre los pacientes con depresión refractaria. En 1993, analizando seis estudios clínicos, Howland encontró que el $52 \%$ de los pacientes con depresión refractaria padecían de un hipotiroidismo subclínico. Este autor concluía en el sentido de considerar la existencia de un subtipo biológico de depresión. En 1999, Konig et al. encontraron autoanticuerpos en un alto porcentaje ( $70 \%$ de etre 144$)$ entre pacientes hospitalizados por padecer cuadros depresivos graves, señalando como la hipotireosis puede ser un factor de riesgo para la depresión y para la posible falta de respuesta al tratamiento médico. Una posible relación entre los anticuerpos antitiroideos en la encefalopatía de Hashimoto y la depresión ha sido puesto de manifiesto en el mas reciente estudio de Rolland y Chevrollier (2001).

De acuerdo con el estudio de Palinkas et al. (2001), los síntomas relacionados con los trastornos del humor en el invierno reflejan la existencia de un cierto estado de relativo hipotiroidismo. 
Por otro lado, los resultados del estudio de Vandoolaeghe, en 1997, no dan evidencia de que el hipotiroidismo subclínico aparezca mas que de una forma coincidente entre los pacientes depresivos tratados por su cuadro resistente.

Es más, Fava et al. en 1995 señalan como entre sus pacientes depresivos ambulatorios la presencia de hipotiroidismo o de hipertiroidismo es algo verdaderamente infrecuente. Lo mismo señalaron Ordas y Lávate en 1995, quienes no hallaron enfermedades tiroideas importantes entre sus pacientes hospitalizados por depresión. Lingjaerde et al. tampoco encontraron una correlación significativa entre niveles hormonales y gravedad de los síndromes depresivos invernales (1995).

Nos gustaría que nuestro trabajo pudiera contribuir a un mejor diagnóstico y tratamiento de los cuadros depresivos. Como decíamos anteriormente, el hipotiroidismo puede contribuir al desarrollo de un cuadro depresivo. Asumimos el hecho de que un paciente con hipotiroidismo y con una predisposición biológica a la depresión probablemente padecerá depresión. Desde el momento en que los pacientes son tratados de su hipotiroidismo las manifestaciones depresivas quedarán enmascaradas, atribuyéndolas a la enfermedad tiroidea. Ello conlleva la posibilidad de no ser tratados como depresivos con el riesgo consiguiente de la encronización de su trastorno depresivo. Por otro lado, nuestros datos nos permiten decir que la mitad de las depresiones refractarias contribuyen al hipotiroidismo. Así que los aspectos psicológicos, psiquiátricos y psicosociales de las enfermedades endocrinas en general no deberían ser olvidados (Sonino 1997).

\section{Conclusiones}

Dos tercios de los pacientes con disfunción tiroidea padecen de trastornos depresivos clínicamente significativos. Los trastornos depresivos se hallan mas representados entre los pacientes con hipotiroidismo que entre los hipertiroideos.

Los trastornos depresivos entre los pacientes con disfunción tiroidea fueron menos graves que entre los pacientes con Depresión Mayor Unipolar.

Los pacientes con hipertiroidismo tenían menos síntomas graves del tipo de la inhibición que los pacientes con Depresión Mayor.

Los síntomas graves de agitación entre los pacientes con Depresión Mayor Unipolar son significativamente diferentes de los síntomas presentados por los pacientes con hipotiroidismo.

Existe una prevalencia de síntomas de agitación entre los pacientes con hipertiroidismo y una dominancia de síntomas inhibitorios depresivos entre los pacientes con hipotiroidismo.

La relativa alta representación de los trastornos depresivos entre los pacientes con disfunción tiroidea los convierte en un grupo de riesgo potencial. Este hecho es importante tenerlo en cuenta en lo referente al diagnóstico y al tratamiento, ya que nos ayudará a prevenir un posible riesgo en cuanto a la cronicidad de la depresión.

\section{Bibliografía}

AMSTERDAM, J.D., FAVA, M., MAISLIN, G., ROSENBAUM, J., HORNIG-ROHAN, M. TRH stimulation test as a predictor of acute and long-term antidepres- 
sant response in major depression. J. Affect. Disord. 38 (23): 165-172, 1996.

BARTALENA, L., PLACIDI, G.F., MARTINO, E. et al. Nocturnal serum thyrotropin (TSH) surge and the TSH response to TRH-releasing hormone: dissociated behaviour in untreated depressives. J. Clin. Endocrinol. Met., 71 (3): 650-655, 1990.

BAUER, M.S., DROBA, M., WHYBROW, P.C. Disorders of the thyroid and Parathyroid, 41-70. Nemeroff, C.B., Loosen, P.T., (eds.) Handbook of clinical psychoneuroendocrinology. Chichester-New York-Brisbane-TorontoSingapore John Wiley and Sons, 1987.

CANCRO, R. Overview of affective disorders, 760763. Kaplan, H.I., Sadock, B.J., (eds.) Comprehensive textboock of psychiatry/IV. 4. Rev. Baltimore/London: Williams and Wilkins Co. 1985.

CHECKLEY, S. Thyrotoxicosis and the course of manicdepressive illness. Brit J Psychiatry, 133: 219-23, 1978.

Clinical Society of London. (1888). Report on myxedema. Transactions of the Clinical Society of London, 21 (Suppl.), 1X.

COPOLOV, D.L., RUBIN, R.T. Endocrine disturbances in affective disorders and schizophrenia, 160-194. Nemeroff CB, Loosen PT, (eds.). Handbook of clinical psychoneurændocrinology. Chichester-New York-BrisbaneToronto-Singapore: John Wiley and Sons, 1987.

COWDRAY, R.W., WEHR, T.A., ZIS, A.P., GOODWIN, F.K. Thyroid abnormalities associated with rapid-cycling bipolar illness. Arch. Gen. Psychiatry, 40: 414-420, 1983.

DANIELS, G.H., MARTIN, J.B. Neuroendocrine regulation and disease of the anterior pituitary and hypothalamus, 1655-1679. Wilson, J.D., Braunwald, E., Isselbacher, K.J., Petrsdorf, R.G., Martin, J.B., Fauci, A.S., Root, R.K.(eds.). Harrison's principles of internal medicine. 12. rev., vol 2. New York, St. Louis, San Francisco, Colorado Springs, Auckland, Bogota, Caracas, Hamburg, Lisbon, London, Madrid, Mexico, Milan, Montreal, New Delhi, Paris, San Juan Sao Paulo, Singapore, Sydney, Tokyo, Toronto: Mc Grow-Hill, Inc, 1991.

DENICOFF, K.D, JOFFE, R.T., LAKSHMANAN, M.C., ROBBINS, J.R., RUBINOW, D.R. Neuropsychiatric manifestations of altered thyroid state. Am. J. Psychiatry., 147 (1): 94-99, 1990.

Diagnostic and Statistical Manual of Mental Disorders. 4. rev. American Psychiatric Association. Washington, D.C., 1994.

DORN, L.D., BURGESS, E.S., DICHEK, H.L., PUTNAM, F.W., CHROUSOS, G.P., GOLD, P.W. Thyroid hormone concentrations in depressed and non depressed ado- lescents: group differences and behavioral relations. J. Am. Acad. Child Adolesc. Psychiatry, 35 (3): 299-306, 1996.

DUVAL, F., MOKRANI, M.C., BAILEY, P., CORREA, H., DIEP, T.S., CROCQ, M.A., MACHER, J.P. Thyroid axis activity and serotonin function in major depressive episode. Psychoneuroendocrinology, 24 (7): 695-712, 1999.

EARLE, B. Thyroid hormone and tricyclic antidepressants in treatment-resistant depression. Am. J. Psychiatry, 126: 1667-1669, 1970.

EXTEIN, L., POTTASH, A.L.C., GOLD, M.S., SILVER, J.M. Thyroid stimulating hormone response in unipolar depression before and after clinical improvement. Psychiatry Res, 6: 161-169.1982.

FAVA, M., LABBATE, L.A., ABRAHAM, M.E., ROSENBAUM, J.F. Hypothyroidism and hyperthyroidism in major depression revisited J. Clin. Psychiatry, 56 (5): 186-192, 1995.

GOLD, P.W., GOODWIN, F.K., CHROUSOS, G.P. Clinical and biochemical manifestations of depression. Relation to the neurobiology of stress. New England J. Med., 319 (7): 413-419, 1989.

GOLD, M., POTTASH, A., EXTEIN, I. Hypothyroidism and depression: Evidence from complete thyroid evaluation. JAMA, 245: 1919-1921, 1981.

HAGGERTY Jr., J.J., STERN, R.A., MASON, G.A., BECKWITH, J., MORCY, C.E. PRANGE Jr., A.J. Subclinical Hypothyroidism: a modifiable risk factor for depression? Am. J. Psychiatry, 150 (3): 508-510, 1993.

HAMILTON, M. 049 HAMD. Hamilton depression scale, 179-192. Guy W, (ed.) ECDEU Assesment Manual for Psychopharmacology. Rev. Ed. Rockville, Maryland, 1976.

HOFMANN, P., SCHROTTER, K.H. Hypophyscalhypothalamo-thyroid axis in affective disorders. Acta Med. Austriaca, 26 (4):123-125, 1999.

HOWLAND, R.H. Thyroid dysfunction in refractory depression: implications for pathophysiology and treatment J. Clin. Psychiatry, 54: 47-54, 1993.

JACKSON, I. Thyrotropin-releasing hormone (TRH): Distribution in mammalian species and its functional significance, 3-8. Griffits, E.C., Bennett, G.W. (eds.) Thyrotropin-releasing hormone. New York: Raven Press, 1983.

JAIN, V. A psychiatric study of hypothyroidism. Psychiatr. Clin., 5: 121-130, 1972.

JOFFE, R.T., BAGBY, R.M., LEVITT, A.J. The thyroid and melancholia. Psvchiatry Res, 42 (1): 73-80, 1992.

JOFFE, R.T., LEVITT, A.J.: Major depression and subclinical (grade 2) hypothyroidism. Psychoneuroendocrinolo, 17: 215-221, 1992. 
KIRKEGAARD, C., FABER, J., COHN, D., KOLENDORF, K., THOMSEN, H.F. LUMHOLTZ, I.B., SIERSBACK-NIELSEN, K., FRIIS, T. Serum 3'monoiodthyronine levels in normal subjects and in patients with thyroid and non-thyroid disease. Acta Endocrinol, 97: 454-460, 1981.

KIRKEGAARD, C., KARNER, A., FABER, J. Increased production of thyroxine and inappropriately elevated serum thyrotropin leves in endogenous depression. Biol Psychiatry, 27 (5): 472-476, 1990.

KJELMANN, B.F. BECK-FRIIS, J. LJUNGGREN, J.G. WETTERBERG, L. Twenty four-hour serum levels of TSH in affective disorders. Acta Psychiatr. Scand. 69: 491$502,1984$.

KONIG, F., VON HIPPEL, C., PETERSDORFF, T., KASCHKA, W. Thyroid autoantibodies in depressive disorders. Acta Med. Austriaca, 26 (4): 126-128, 1999.

LASSER, R.A., BALDESSARINI, R.J. Thyroid hormones in depressive disorders: a reappraisal of clinical utility. Harv. Rev. Psychiatry, 4 (6): 291-305, 1997.

LINGIAERDE, O., REICHBORN-KJENNERUD, T., HAUG, E. Thyroid function in seasonal affective disorder. J. Affect. Disord., 33 (1): 39-45, 1995.

LOOSEN, P., PRANGE, A. Serum thyrotropin response to thyrotropin-releasing hormone in psychiatric patiens. Am. J. Psychiatry, 139: 405-416, 1982.

LUCKE, C., HEHRMANN, R., VON MAYERSBACH, K., VON ZUR MÜHLENN, A. Studies on circadian variations of plasma TSH, thyroxine and triiodothyronine in man. Acta Endocrinologica, 86: 81-88, 1977.

MAC CRIMMON, D., WALLACE, J., GOLDBERG, W., STREINER, D. Emotional disturbance and cognitive deficits in hyperthyroidism. Psychosom. Med. 41: 31-40, 1979.

MOREAU, X. JEANNINGROS, R., MAZZOLAPOMIETTO, P. Chronic effects of triiodothyronine in combination with imipramine on 5-HT transporter, 5HT(1A) and 5-HT(2A) receptors in adult rat brain. Neuropsychopharmacology, 24 (6): 652-662, 2001.

MUSSELRNAN, D.L., NEMEROFF, C.B. Depression and endocrine disorders: focus on the thyroid and adrenal system. Br. J. Psychiatry Suppl. 30: 123, 1996.

MÜLLER, B., BÖNING, J. Changes in the pituitarythyroid axis accompanying major affective disorders. Acta Psychiatr. Scand., 77: 143-150, 1988.

NAKAMURA, T., NOMURA, J. Comparison of thyroid function between responders and nonresponders to thyroid hormone supplementation in depression. Jpn J. Psychiatry Neurol., 46 (4): 905-909, 1992.
National Institute of Mental Health. 028 CGI. Clinical Global Impressions, 217-22. Guy W. (ed.) ECDEU Assesment Manual for Psychopranacoloy. Rev. Ed. Rockville, Maryland, 1976.

ORDAS, D.M., LABBATE, L.A. Positive screening of thyroid function in patients hospitalised for major depression or dysthymia. Ann. Clin. Psychiatry, 7 (4): 161-165, 1995.

PALINKAS, L.A., REED, H.L., REEDY, K.R., DO, N.V., CASE, H.S., FINNEY, N.S. Circannual pattern of hypothalamic-pituitary-thyroid (HPT) function and mood during extended antartic residence. Psychoneurcendocrinology, 26 (4): 421-431, 2001.

PARRY, C. (1825). Collected Works, Vol. I. London.

PEAKE, R. Recurrent apathetic hyperthyroidism. Arch. Int. Med. 141: 258-262, 1981.

PRANGE, A.J., GARBUT, J.L., LOOSEN, P.T. The hypothalamic pituitary thyroid axis in affective disorders, 629-636. Meltzer, H.Y., (ed) Psychopharmacology: The Third Generation of Progress. New York: Raven Press, 1987.

PRANGE, A.J., LARA, P.P. WILSON, I.C. ALLTOP, L.B. Effects of Thyrotropin-releasing hormone in depression. Lancet. 11: 999-1002, 1972.

PRANGE, A.J. JR, WILSON, I.C., LYNN, C.W. et al. L-Tryptophan in mania: contribution to a permissive hypothesis of affective disorder. Arch. Gen. Psychiatry, 30: 56-62. 1974.

PRANGE, A., WILSON, I., RABON, A., LIPTON, M. Enhancement of imipramine antidepressant activity by thyroid hormone. Am. J. Psychiatry, 126: 457-460, 1969.

ROCKEY, P., GRIEP, R. Behavioural dysfunction in hyperthyroidism: Improvement with treatment. Arch. Inter. Med. 140: 1194-1197, 1980.

ROLLAND, F., CHEVROLLIER, J.P. Depression, antithyroid antibodies and Hashimoto encephalopathy. Encephale, 27 (2): 137-142, 2001.

RUBIN, R.T., POLAND, R.E., LESSER, I.M., MARTIN, D.J. Neuroendocrine aspects of primary endogenous depression-IV. Pituitary-thyroid axis activity in patients and matched control subjects. Psychoneurcendocrinology, 12 (5): 333-347, 1987.

SARTORIUS, N. Cross cultural research on depression, 6-11. Kielholz, P., Poldinger, N., ed. Latest findings on the aetiology and therapy of depression. Basle: International commemorative symposium organised in collaboration with WHO and WPA. Psychopathology, 19 (suppl. 2), 1986. 
SCHILDKRAUT, J.J. The catecholamine hypothesis of affective disorders: A review of supporting evidence. Am. J. Psychiatry, 122: 509-522, 1965.

SCHILDKRAUT, J.J., GREEN, A.I., MOONEY, J.J. Affective disorders: Biochemical Aspects, 769-778. Kaplan, H.I., Sadock, B.J., (eds.) Comprehensive textbook of psychiatry/IV. 4. rev. Baltimore/London: Williams and Wilkins Co, 1985.

SCHMAUSS, M. Biological hypothesis in depression research Psychiatlia Daoubina, 4: 37-48, 1992.

SONINO, N. From the lesson of Harvey Cushing to current knowledge: psychosocial aspects of endocrine disease (editorial). Psychother Psychosom, 66 (3): 113, 1997.

THOMASTON, J., MÄNNISTÖ, P. Neurotransmitter regulation of anterior pituitary hormones. Pharmacological Review, 37 (3): 249-332. 1985.

VAN DER KAR, L.D. Neuroendocrine aspects of the serotonergic hypothesis of depression. Neurosci Biobehav. Rev., 13 (4): 237-246. 1989.

VILLANI, S., WERTZEL, W. Secondary mania (letter). Arch Gen Psychiatry, 36: 1031, 1979.

VANDOOLAEGHE, E., MAES, M., VANDEVYVERE, J., NEELS, H. Hypothalamic-pituitary thyroid axis function in treatment resistant depression. J. Affect. Disord., 43 (2): 143-150. 1997.

WARTOTSKY, L., INGBAR, S.H. Diseases of the thyroid, 1692-712. Wilson, J.D., Braunwald, E., Isselbacher, K.J., Petrsdorf, R.G., Martin, J.B., Fauci, A.S., Root, R.K., (eds.) Harrison's principles of internal medicine. 12. rev., Vol. 2. New York St Louis, San Francisko. Colorado
Springs, Auckland, Bogotá, Caracas, Hamburg, Lisbon. London, Madrid Mexico, Milan, Montreal, New Delhi, Paris, San Juan, Sao Paulo, Singapore. Sydney. Tokyo. Toronto: Mc Grow-Hill. Inc. 1991.

WENZEL, K., MEINHOLD, H., RAFFENBERG, M., ADLKOFFER, F., SCHLEUSENER, H. Classification of hypothireoidism in evaluating patients after radioactive therapy by serum cholesterol, T3 uptake, total T4, FT4index, total T3, basal TSH and TRH test. Eur. J. Clin. Invest. 4: 141-48, 1974.

WHYBROW, P.C. PRANGE, A.J. JR. A hypothesis of thyroid-catecholamine-receptor interactions. Arch. Gen. Psychiatry, 38: 106-113, 1981.

WHYBROW, P., PRANGE, A., TREADWAY, C. Mental changes accompanying thyroid gland dysfunction. Arch. Gen. Psychiatry, 20: 48-62, 1969.

WILSON, W., JOHNSON, J., SMITH R. Affective changes in thyrotoxicosis and experimental hypermetabolism. Recent Adv. Biol. Psychiatry 4: 234-242, 1962.

ZUNG, W.W.K. A Self Rating Depression Scale. Arch. Gen. Psychiatry, 12: 63-70, 1965.

Dirección para correspondencia: Ljiljana Radanović-Grgurić Psychiatric Clinic Osijek Hospital Clinic Huttlerova 4 31000 Osijek CROACIA 